\title{
Drug-induced eosinophilic pneumonia in a patient with Crohn's disease: diagnosis and treatment using fraction of exhaled nitric oxide
}

\author{
Jina Yeo, Hyun Sun Woo, Sang Min Lee, Yoon Jae Kim, Kwang An Kwon, Dong Kyun Park, Jung Ho Kim, \\ Kyoung Oh Kim, Jun-Won Chung \\ Department of Internal Medicine, Gachon University Gil Medical Center, Incheon, Korea
}

Oral 5-aminosalicylic acid agents (mesalazine and sulfasalazine) and azathioprine are the mainstays of treatment for inflammatory bowel disease. Reports of pulmonary toxicity induced by oral 5-aminosalicylic acid agents or azathioprine in patients with inflammatory bowel disease are very rare; to date, only 38 cases have been reported worldwide. We, herein, report a case involving a 26-year-old man who was diagnosed with eosinophilic pneumonia after using mesalazine and azathioprine for the treatment of Crohn's disease and recovered after treatment. We also found that the fraction of exhaled nitric oxide level was elevated in this patient. After treatment, the fraction of exhaled nitric oxide level decreased and the symptoms improved. The present case shows that fraction of exhaled nitric oxide is related to the disease activity and treatment effectiveness of druginduced eosinophilic pneumonia. (Intest Res 2017;15:529-534)

Key Words: Pulmonary eosinophilia; Mesalamine; Azathioprine; Crohn disease; Nitric oxide

\section{INTRODUCTION}

Oral 5-aminosalicylic acid (5-ASA) agents such as mesalazine and sulfasalazine are the mainstay of treatment for mild to moderate IBD. Although sulfasalazine is an effective treatment for IBD, up to $30 \%$ of patients experience adverse effects such as nausea, vomiting, abdominal discomfort, diarrhea, headache, pancreatitis, agranulocytosis, and pulmonary toxicity. The newer sulfa-free 5-ASA agent, mesalazine, delivers increased amounts of the active compound while limiting the adverse effects mostly caused by sulfapyridine. ${ }^{1}$ Azathioprine (AZA) is widely used in the treatment of IBD; this drug can induce and maintain remission with a re-

Received August 4, 2016. Revised November 6, 2016. Accepted November 21, 2016. Published online February 27, 2017

Correspondence to Jun-Won Chung, Division of Gastroenterology, Department of Internal Medicine, Gachon University Gil Medical Center, 21 Namdong-daero 774 beon-gil, Nandong-gu, Incheon 21565, Korea. Tel: +82-32-460-3778, Fax: +82-32-460-3408, E-mail: junwonchung@hanmail.net

Financial support: None. Conflict of interest: None. sponse rate of $55 \%$ to $70 \%{ }^{2}$

Extraintestinal manifestations associated with IBD are seen in $21 \%$ to $41 \%$ of patients with IBD. ${ }^{3}$ Pulmonary disorders occurring as extraintestinal manifestations of IBD are rare. Such pulmonary disorders can be divided into those that develop secondary to IBD itself and those that occur as adverse reactions or toxicities associated with the drugs used to treat IBD. These pulmonary disorders associated with the drugs may be divided into noninfectious pulmonary disease and opportunistic pulmonary infections. ${ }^{4}$

The fraction of exhaled nitric oxide (FENO) is a marker of airway diseases including asthma, eosinophilic inflammation, and bronchial wall inflammation. FENO levels increase in eosinophilic inflammation. Recently, FENO was utilized in some cases such as asthma, inflammatory disease, and druginduced eosinophilic pneumonia. After treatment, FENO levels were reduced. ${ }^{5}$

Reports of drug-induced pulmonary toxicity in patients with IBD are rare; only 38 cases have been described world-

- Copyright 2017. Korean Association for the Study of Intestinal Diseases. All rights reserved.

This is an Open Access article distributed under the terms of the Creative Commons Attribution Non-Commercial License (http://creativecommons.org/licenses/by-nc/4.0)

which permits unrestricted non-commercial use, distribution, and reproduction in any medium, provided the original work is properly cited. 
wide. Domestic reports of drug-induced pulmonary toxicity in patients with IBD include 1 case of eosinophilic pneumonia and 1 case of interstitial pneumonia. Only 2 cases of eosinophilic pneumonia due to mesalazine have been reported in patients with CD. Only 3 cases of AZA-induced eosinophilic pneumonia have been reported worldwide. We, herein, describe a patient with $\mathrm{CD}$ who developed the rare complication of eosinophilic pneumonia during mesalazine and AZA treatment. The patient's disease activity and treatment effectiveness were evaluated with FENO.

\section{CASE REPORT}

A 26-year-old man with a 3-week history of coughing presented to Gachon University Gil Medical Center. He had been diagnosed with iron deficiency anemia 2 years previously. Ten months previously, he underwent small bowel resection and anastomosis for treatment of a small bowel perforation and was diagnosed with CD based on the biopsy findings (inflammation and noncaseating granuloma formation on the bowel wall) (Fig. 1).

The patient was a lifelong nonsmoker and nondrinker and had not recently traveled to any foreign countries. He had no significant family medical history. After the diagnosis of CD, he was treated with mesalazine at $3 \mathrm{~g} /$ day for 10 months. During the first week after the diagnosis, he had received prednisolone at $30 \mathrm{mg} /$ day; this dose was tapered and discontinued. He developed tenesmus and hematochezia after 6 months of treatment with mesalazine, and prednisolone was restarted at $15 \mathrm{mg} /$ day and the dose was tapered for 3 weeks. Four weeks before his visit to our hospital, the hematochezia reappeared along with abdominal pain and an elevated CRP level. Because of disease exacerbation, $50 \mathrm{mg}$ of AZA was added until the day of hospital admission.

The patient's abdominal pain had subsided at the time of hospital admission, but he exhibited dry coughing with white sputum, dyspnea, and chest discomfort. He had no chills or fever. His blood pressure was 110/70 mmHg, pulse was 106/ min, body temperature was $36.5^{\circ} \mathrm{C}$, and peripheral capillary oxygen saturation was $97 \%$. He had clear consciousness but exhibited a sickly appearance. Both the right and left lung fields were clear during chest auscultation. Peripheral blood test results revealed the following: white blood cell count, 7,020/ $\mathrm{mm}^{3}$ (neutrophils, $60.7 \%$; eosinophils, $7.4 \%$ ); hematocrit, $10.8 \mathrm{~g} / \mathrm{dL}$; and platelet count, $784,000 / \mathrm{mm}^{3}$. The blood chemistry results were as follows: serum total protein, $7.7 \mathrm{~g} /$ $\mathrm{dL}$; albumin, $4.1 \mathrm{~g} / \mathrm{dL}$; AST, $13 \mathrm{U} / \mathrm{L}$; alanine transaminase, 12 $\mathrm{U} / \mathrm{L} ; \mathrm{BUN}, 11.9 \mathrm{mg} / \mathrm{dL}$; creatinine, $0.7 \mathrm{mg} / \mathrm{dL}$; and CRP, 7.09 $\mathrm{mg} / \mathrm{dL}$. A chest radiograph acquired at the time of admission showed signs of subpleural consolidation and suspicious cystic lesions in both lungs (possible pneumonia) (Fig. 2). Chest CT showed subpleural consolidation and groundglass opacities in the bilateral lung fields (Fig. 3A). Similar findings were present on small bowel CT performed 3 weeks before admission ( 1 week into AZA treatment); however, these findings were more severe.

Because of signs of atypical pneumonia and elevated eosinophil count of $519.5 / \mathrm{mm}^{3}$, the possibility of eosinophilic pneumonia was considered, and mesalazine and AZA were discontinued. Findings of sputum AFB smear, fungal smear,
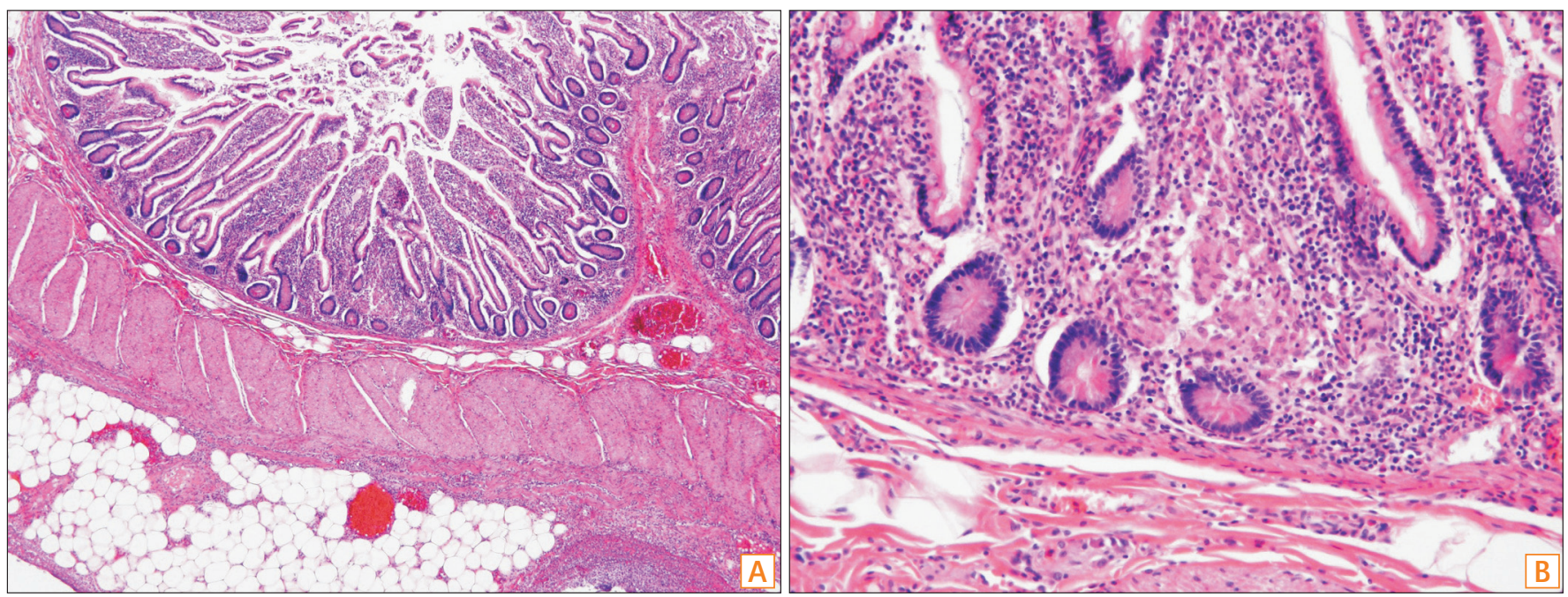

Fig. 1. Histologic examination shows inflammation and noncaseating granulomas on the bowel wall $(A, H \in E$, magnification $\times 40 ; B, H \& E$, magnification $\times 200$ ). 
bacterial culture, and cytological examination, and results of QuantiFERON Gold test, virology test, Chlamydia IgM test, Mycoplasma antibody test, urine Streptococcus pneumoniae antigen test, and urine Legionella antigen test were all negative. Although an Aspergillus antigen test result was positive, it was deemed false-positive because of treatment with piperacillin/tazobactam. Bronchoscopic findings were negative, and bronchoalveolar lavage (BAL) showed a white blood cell count of $78 / \mathrm{mm}^{3}$ (eosinophils, $14 \%$ ) and a FENO of 74 parts per billion (ppb) (Fig. 4). Results of AFB test, fungal and bacterial cultures, tuberculosis test, respiratory virus antigen test, cytomegalovirus PCR, and Epstein-Barr virus PCR were all negative.

Mesalazine was continued for 10 months. A chest radiograph after 1 week of AZA treatment showed a reticulonod-

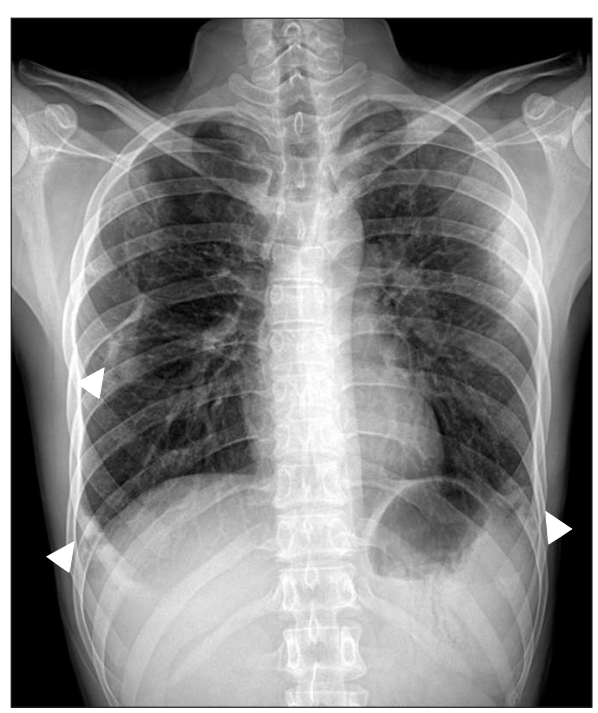

Fig. 2. A chest radiograph on admission shows subpleural consolidation and cystic lesions (arrowheads) in both lungs.
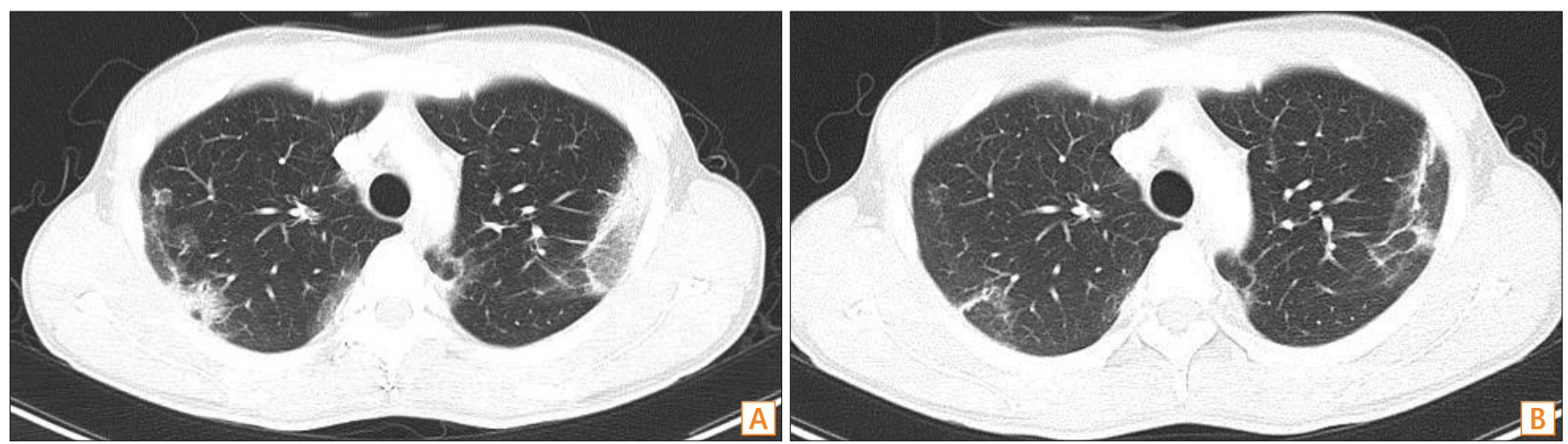

Fig. 3. Chest CT shows subpleural consolidation and ground-glass opacities in the bilateral lung fields on admission (A) and follow-up chest CT after 5 days showed improvement (B). ular density and peripheral blood tests showed increased eosinophils; BAL also showed increased eosinophils (14\%). Based on these results, the patient was diagnosed with mesalazine- or AZA-associated eosinophilic pneumonia. After discontinuation of mesalazine and AZA and 2 weeks of treatment with prednisolone at $30 \mathrm{mg} /$ day, all pulmonary symptoms of the patient resolved; follow-up chest CT after 5 days also showed improvement (Fig. 3B). Blood test results during outpatient visits showed decreased eosinophil levels (as low as 3.2\%) and a decreased posttreatment FENO level (13 ppb) on BAL; chest radiographs no longer showed signs of pulmonary abnormalities. The patient's CD stabilized even after the discontinuation of mesalazine, and the eosinophilic pneumonia did not recur.

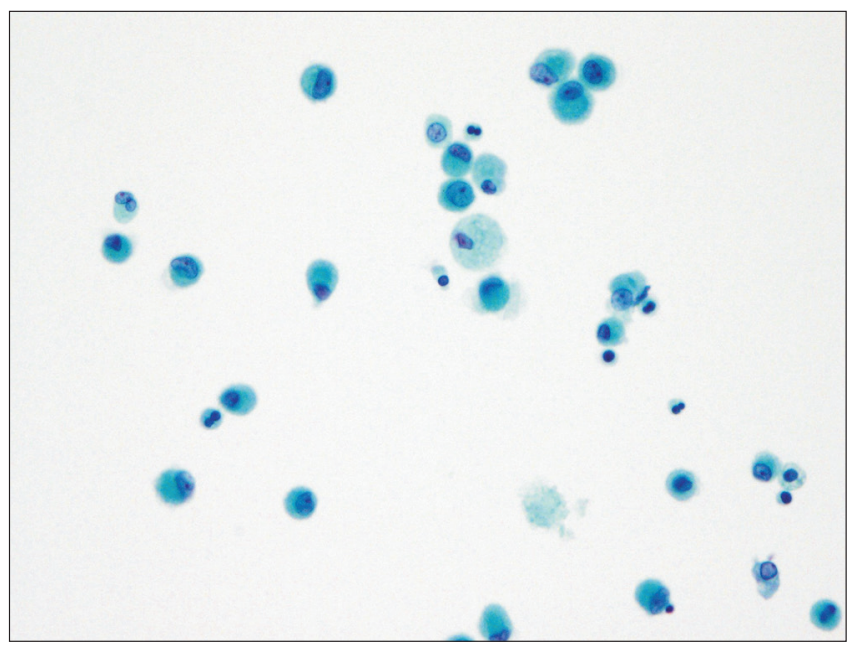

Fig. 4. Bronchial alveolar washing and cytology shows eosinophils (magnification, $\times 400)$. 


\section{DISCUSSION}

Our patient had a 1-year history of $\mathrm{CD}$, and his pulmonary symptoms occurred 10 months after the initial administration of mesalazine and after 1 week of AZA treatment. Multifocal subpleural consolidations and ground-glass opacities in both the left and right basal lung fields were present on the initial chest radiograph and chest CT. BAL was performed for a definitive diagnosis. Eosinophilia was detected in the patient's peripheral blood (7.4\%) and BAL fluid (14.0\%). His clinical signs and radiologic findings improved after discontinuation of mesalazine and AZA and the 2-week steroid treatment. The FENO was 74 ppb on BAL at diagnosis, decreasing to $13 \mathrm{ppb}$ after treatment.

The inflammatory status of acute eosinophilic pneumonia (AEP) may be reflected in the FENO level, which has been shown to be higher in patients with than in those without AEP. In addition, the FENO level has been shown to decrease with steroid treatment. ${ }^{5}$ Because our patient's sputum AFB smear test, fungal smear test, bacterial culture, cytology, QuantiFERON Gold test, and virology test results were all negative, we excluded infectious disease and a diagnosis of mesalazine- or AZA-induced eosinophilic pneumonia was made. His pulmonary symptoms resolved after treatment with prednisolone at $30 \mathrm{mg} /$ day for 2 weeks, and follow-up chest CT 5 days later showed improvement. Blood test results from his outpatient visits showed decreased eosinophil levels, and chest radiographs no longer showed signs of pulmonary abnormalities. A drug lymphocyte stimulation test and re-challenge were performed to confirm the presence of drug-induced eosinophilic pneumonia. However, we did not perform a mesalazine or AZA lymphocyte stimulation test because the patient's condition may have become aggravated. If mesalazine or AZA is inevitably needed to treat IBD, oral desensitization to 5-ASA should be performed. ${ }^{6}$ These reactions can also be overcome by desensitization of the immune system to the drug.

AEP is a distinct pneumonia syndrome. The etiology and pathophysiology of AEP are unclear; however, AEP is often associated with drug treatment. ${ }^{8}$

Sulfasalazine (sulfapyridine bound to 5-ASA) and mesalazine (5-ASA alone) are treatment options for patients with variations of IBD such as CD and UC. ${ }^{1}$ AZA, a purine ana$\log$, induces and maintains remission in IBD. ${ }^{9}$ The detailed mechanisms of sulfasalazine action remain unknown, potential mechanisms include scavenging of free radicals, inhibition of nuclear factor $\kappa \mathrm{B}$, induction of apoptosis, and interference with arachidonic acid metabolism., ${ }^{4,10}$ Sulfasalazine is metabolized to sulfapyridine and 5-ASA. Adverse effects of sulfasalazine include nausea, gastric discomfort, headache, anorexia, skin rashes, male infertility, and pulmonary syndromes during IBD treatment. These adverse effects of sulfasalazine are caused by its sulfamic vector, not by 5 -ASA or mesalazine. ${ }^{11}$ 5-ASA clinically decreases inflammation of the colon and rectum. In this regard, mesalazine has been widely used for IBD treatment. Possible mechanisms of action of mesalazine include the inhibition of interleukin- 1 , tumor necrosis factor- $\alpha$, and nuclear factor $\kappa \mathrm{B}$. However, the pathogenesis of mesalazine-related pulmonary involvement remains unclear. Four proposed mechanisms are oxidative injury by drugs such as nitrofurantoin, direct cytotoxic effect on alveolar capillary endothelial cells, cell-mediated injury secondary to deposition of phospholipids, and immunemediated injury resulting in clinical symptoms of systemic lupus erythematosus. Immune-related alveolitis secondary to lymphocyte stimulation is currently being considered a possible mechanism of mesalazine-related pulmonary in-

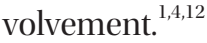

AZA is a purine analog inhibiting the synthesis of purine nucleotide. The pathogenesis of AZA is not well understood. There are 2 alternative pathways; one to 6-thiouric acid mediated by xanthine oxidase and the other to 6-methylmercaptopurine mediated by thiopurine methyltransferase. ${ }^{13}$ AZA is frequently associated with a number of adverse effects, and it is usually discontinued due to the adverse effects. There are 2 types of adverse effects: (1) the allergic type including pancreatitis, fever, rash, malaise, nausea, diarrhea, and hepatitis, and the (2) the nonallergic type including leucopenia and some forms of hepatitis. ${ }^{14}$

The pulmonary adverse effects of mesalazine and AZA include fever, fatigue, dyspnea, chest pain, and coughing. ${ }^{15}$ The adverse effects of mesalazine appear 1 to 6 months after treatment. The adverse effects of AZA appear within 1 month after treatment. If interstitial infiltration with a ground-glass pattern and pulmonary nodules are present on a chest radiograph or CT scan, peripheral eosinophilia may be detected on laboratory tests. BAL fluid may show elevated eosinophil and lymphocyte counts and a decreased CD4/CD8 ratio. Pulmonary function tests usually reveal a reduced diffusion capacity for carbon monoxide. Histological findings include eosinophilic infiltration of the alveoli, lymphocytic infiltration of the interstitium, and nonnecrotizing granulomas. ${ }^{12}$ It is not easy to distinguish drug-related pulmonary involvement from pulmonary manifestations of IBD. Mesalazine affects the lung parenchyma, while IBD affects the large airways of the lung. ${ }^{16,17}$ Mesalazine-induced 
lung disease includes eosinophilic pneumonia and fibrosing alveolitis. AZA can lead to opportunistic infections.

To date, 38 cases (39 patients) of mesalazine-related pulmonary involvement have been reported worldwide. Among them, eosinophilic pneumonia was the most common presentation. ${ }^{3}$ Thirty-three patients had UC (84.6\%), and 6 patients had CD (15.4\%). At the time of symptom development, the mean age of mesalazine-related pulmonary involvement was 42 years. The relationship between the dose of mesalazine and the lung toxicity was unclear. The dose of mesalazine varied from $750 \mathrm{mg} /$ day to $4800 \mathrm{mg}$ /day. The interval between the start of mesalazine treatment and the expression of pulmonary symptoms varied from 2 days to $4-5$ years. In total, 38 patients stopped mesalazine, and 23 of them (60.5\%) were treated with systemic glucocorticoids. Interstitial lung infiltration was the most common radiologic finding (73.0\% of patients). Seven patients underwent a rechallenge, and 4 (57.1\%) developed recurrent pulmonary involvement. ${ }^{18}$ AZA-induced pulmonary toxicity has rarely been reported in patients with IBD. AZA-related pulmonary toxicity was first reported by Rubin et al. ${ }^{19}$ in 1972, with the report describing a CD patient with exertional dyspnea. Krowka et al. ${ }^{20}$ in 1983 reported in a 35-year-old man with intermittent fever. AZA-related pulmonary toxicity was mostly reported in patients with renal allograft transplantation. In IBD patients, AZA-related pulmonary toxicity has rarely been reported. ${ }^{14}$

The present study had 2 main limitations. First, we were unable to elucidate the mechanism of the mesalazine- or AZA-induced eosinophilic pneumonia. Second, re-challenge with mesalazine and AZA was not performed because the patient did not have symptoms of IBD. Mesalazine-induced eosinophilic pneumonia usually occurs after 2 to 6 months of treatment. In some case reports, this varied from a few days to years after treatment. AZA induced pulmonary toxicity occurs within 1 month after initiation of treatment for IBD. In IBD patients, AZA-induced eosinophilic pneumonia has rarely been reported. Our patient had a 1-year history of $\mathrm{CD}$, and his pulmonary symptoms occurred 10 months after the initial administration of mesalazine and only after 1 week of AZA treatment. In this regard, the AZA was more likely related to the eosinophilic pneumonia than to mesalamine, considering the time interval between drugs. Ideally, AZA re-challenge would be a definitive diagnostic tool. However, this seemed unethical because the patient has no symptoms to date.

Despite these limitations, this is the first report of eosinophilic pneumonia in a patient treated with mesalazine and AZA for CD. We also found that the FENO level was elevated in this patient with drug-induced eosinophilic pneumonia. After treatment of the drug-induced eosinophilic pneumonia, the FENO level decreased and the symptoms improved. In this regard, the FENO level might be related to the disease activity and treatment effectiveness of drug-induced eosinophilic pneumonia.

In conclusion, we have described a 26-year-old man who was diagnosed with eosinophilic pneumonia after treatment with mesalazine and AZA for CD and who recovered after treatment. Additionally, the present study shows that the FENO is related to disease activity and treatment effectiveness of drug-induced eosinophilic pneumonia.

\section{REFERENCES}

1. Tanigawa K, Sugiyama K, Matsuyama H, et al. Mesalazineinduced eosinophilic pneumonia. Respiration 1999;66:69-72.

2. Fangbin Z, Xiang G, Liang D, et al. Prospective evaluation of pharmacogenomics and metabolite measurements upon azathioprine therapy in inflammatory bowel disease: an observational study. Medicine (Baltimore) 2016;95:e3326. doi: 10.1097/ MD.0000000000003326.

3. Storch I, Sachar D, Katz S. Pulmonary manifestations of inflammatory bowel disease. Inflamm Bowel Dis 2003;9:104-115.

4. Ahnfelt-Rønne I, Nielsen OH, Christensen A, Langholz E, Binder V, Riis P. Clinical evidence supporting the radical scavenger mechanism of 5-aminosalicylic acid. Gastroenterology 1990;98(5 Pt 1):1162-1169.

5. Lee JE, Rhee CK, Lim JH, et al. Fraction of exhaled nitric oxide in patients with acute eosinophilic pneumonia. Chest 2012;141: 1267-1272.

6. Stein RB, Hanauer SB. Comparative tolerability of treatments for inflammatory bowel disease. Drug Saf 2000;23:429-448.

7. Korelitz BI, Reddy B, Bratcher J. Desensitization of patients with allergic reactions to immunosuppressives in the treatment of inflammatory bowel disease. Expert Opin Drug Saf 2010;9:379-382.

8. Allen JN. Drug-induced eosinophilic lung disease. Clin Chest Med 2004;25:77-88.

9. Sandborn W, Sutherland L, Pearson D, May G, Modigliani R, Prantera C. Azathioprine or 6-mercaptopurine for inducing remission of Crohn's disease. Cochrane Database Syst Rev 2000;(2):CD000545. doi: 10.1002/14651858.CD000545.

10. Bantel H, Berg C, Vieth M, Stolte M, Kruis W, Schulze-Osthoff K. Mesalazine inhibits activation of transcription factor NFkappaB in inflamed mucosa of patients with ulcerative colitis. Am J Gastroenterol 2000;95:3452-3457. 
11. Sullivan SN. Sulfasalazine lung: desensitization to sulfasalazine and treatment with acrylic coated 5-ASA and azodisalicylate. J Clin Gastroenterol 1987;9:461-463.

12. Foster RA, Zander DS, Mergo PJ, Valentine JF. Mesalaminerelated lung disease: clinical, radiographic, and pathologic manifestations. Inflamm Bowel Dis 2003;9:308-315.

13. Fraser AG, Orchard TR, Jewell DP. The efficacy of azathioprine for the treatment of inflammatory bowel disease: a 30 year review. Gut 2002;50:485-489.

14. Ananthakrishnan AN, Attila T, Otterson MF, et al. Severe pulmonary toxicity after azathioprine/6-mercaptopurine initiation for the treatment of inflammatory bowel disease. J Clin Gastroenterol 2007;41:682-688.

15. Schleiermacher D, Hoffmann JC. Pulmonary abnormalities in inflammatory bowel disease. J Crohns Colitis 2007;1:61-69.
16. Camus P, Piard F, Ashcroft T, Gal AA, Colby TV. The lung in inflammatory bowel disease. Medicine (Baltimore) 1993;72:151183.

17. Levine JS, Burakoff R. Extraintestinal manifestations of inflammatory bowel disease. Gastroenterol Hepatol (N Y) 2011;7:235241.

18. Ferrusquía J, Pérez-Martínez I, Gómez de la Torre R, et al. Gastroenterology case report of mesalazine-induced cardiopulmonary hypersensitivity. World J Gastroenterol 2015;21:40694077.

19. Rubin G, Baume P, Vandenberg R. Azathioprin nd acute restrictive lung disese. Aust N Z J Med 1972;2:272-274.

20. Krowka MJ, Breuer RI, Kehoe TJ. Azathioprine-associated pulmonary dysfunction. Chest 1983;83:696-698. 